\title{
A New Theoretical Approach to Allostery
}

\author{
Giovanni Villani* \\ Istituto di Chimica dei Composti OrganoMetallici (UOS Pisa) - CNR, Italy \\ *Corresponding author: Giovanni Villani, Istituto di Chimica dei Composti Organo Metallici (UOS Pisa) - CNR, Area della Ricerca di \\ Pisa, Via G Moruzzi, 156124 Pisa, Italy.
}

To Cite This Article: Giovanni Villani, A New Theoretical Approach to Allostery. Am J Biomed Sci \& Res. 2021 - 12(3). AJBSR.MS.ID.001760.

DOI: $10.34297 /$ AJBSR.2021.12.001760.

Received: 眥 April 01, 2020; Published: 阱 April 08, 2021

\section{Minireview}

Allosteric process isn't new, but recently it has acquired even greater application prominence. In a recent review Exploring allostery's advantages on this phenomenon, [1] (C\&EN Chicago) says "drug hunters are increasingly turning their attention to allosteric sites - the often tough-to-find regulatory pockets. A crop of allostery-focused biotech has emerged to illuminate proteins' hidden sites with a combination of better experimental and computational tools". By a theoretical point of view, moreover, the concept of allostery can also assume a larger definition. For a generic macromolecule, allostery can be defined as the alteration of a property in its active site due to a perturbation generated in its sensor (allosteric) site. Following this definition, hence, at the normal way of identifying the "active" and the "allosteric" sites of a macromolecule, we must add the primary question of characterizing the movement of this perturbation in the macromolecule.

This has two consequences:

a) first of all, it is necessary considering the allosteric phenomenon in a time-dependent way.

b) it is important to explain how it is possible a selectivity between two distant sites of the macromolecule in a very complex system with a very large number of states. Both topics have been considered by us with the help of a quantum approach.

This is the first time that allostery has been studied by Quantum Mechanics (QM). Two are the reasons why we have decided to apply QM to this phenomenon. First of all, it is well-known that the correct mechanical theory for studying the molecular world is QM; nevertheless, at the moment, only Classical Mechanics theory has been applied to the study of allostery from a mechanical point of view. Probably, this is due to the idea that these kinds of systems are too complicated for a quantum study. We believe that the situation has now changed. The second and most important reason is the following. The theoretical study of allostery has been performed with Thermodynamics or Classical Mechanics in literature, but both theories cannot explain the coherent propagation of the perturbation created in the allosteric site, within the macromolecule. In fact, a perturbation generated in a part of the macromolecule, free to move, should be spread everywhere, diluting itself in the countless degrees of freedom of this large molecule. On the contrary, it is well-known that in the allosteric process the perturbation starts from the sensor site and reaches the target site through preferential paths with great efficiency. Since the coherent movement of a perturbation in the macromolecule is essential for the allosteric process, we can assume that the allosteric phenomenon has essential quantum components.

QM can give a significant contribution to the study of this phenomenon in two ways. The first way is a detailed study of a specific molecular system that presents allostery, but this can be done with an accurate quantum mechanical method only for those systems that are not too large, that is to say until about a thousand of atoms. Moreover, this kind of study does not allow an easy generalization of the specific case in order to find general principles on the allosteric process. The second use of QM in the study of allostery may be to derive general modelling rules for this process. In our paper, we have studied allostery following this second way [2].

In our modelling, the macromolecule has been described as a network of nodes (or sites) and edges. Starting from a perturbation generated in the allosteric (or sensor) site, we have studied, 
through QM, how it moves in the network and arrives to the active site. According to us, the sites of the system can represent either the single amino acid of a protein or, more correctly from a modelling perspective, a larger piece of the protein sufficiently isolated and unitary to be considered a site (a protein domain, for example). Each site of the macromolecular network can be described with one or more states, which define its characteristics. In our model each edge is a number that measures the coupling between the nodes.

This numerical value of the coupling between two nodes can be considered constant along the movement of the perturbation or time-dependent and, in this last case, it can change in two different ways:

a. Randomly or

b. As a function of the fraction of the perturbation wave present in the site.

In order to quantify the allosteric phenomenon, the perturbation wave has been characterized by three parameters in the active site:

a. The characteristic time needed to reach this target.

b. The amplitude of the perturbation wave and

c. Its spectral broadening. One of the aims of our work has been to find general rules able to connect these parameters to the characteristics of the system: numbers of sites, energies and site couplings.

Here, we would like to underline that QM allows to consider systems with a very large number of coupled states and, when there are only a few couplings per state, it is possible to consider some hundreds of thousands states with the help of some numerical procedures. From this theoretical study, it is possible to draw some general remarks to deal with a real system that has this process [3]. First of all, it is necessary to identify the macromolecular parts which are involved in this process and that can be schematized as coupled sites. This can be done, as it is now, with molecular mechanics simulations. Once identified the sites, their electronic and vibrational states and their couplings should be calculated with an accurate quantum calculation and through spectroscopic techniques. We should then model the system with a set of coupled sites and find the preferential paths which lead from the allosteric site to the active one; finally, we should follow the dynamics using a quantum approach, where we could consider also the constructive interferences among the different paths that connect the allosteric site to the active one; these interferences could play a decisive role in complex systems with many paths.

A last general question can be posed. If there is such a perturbation that moves along proteins in the allosteric process, is it possible to measure its speed of propagation experimentally? The answer seems to be positive [4]. On the basis of recent pumpprobe-type experiments, a conformational transition, similar to the one occurring upon ligand binding, can be found on a picosecond timescale by a laser pulse. In general, spectroscopic techniques constitute the best way of investigating energy transfer and linking it to signal propagation in proteins. Direct determination of allosteric pathways through spectroscopic methods is therefore currently unfeasible, but we may gain indirect evidence for determining pathways of signal propagation by mutagenesis studies, where mutations at key residues deteriorating the efficiency of energy transport and leading to a loss of biological function.

Finally, we want to spend some words on the more general problem of a complex network of sites with interactions of different types (chemical bonds and weak interactions) among the components. These systems can form both macromolecules and less aggregated entities called supra-systems. In our opinion, also the dynamic of these supra-systems should be studied with Quantum Mechanics. Only a quantum approach allows us to understand the movements within this kind of network weakly bonded and allows us to study the preferential passage of a perturbation among their different sites. The idea that some biological processes, normally intended to function in a classical thermodynamic limit may be able to use states and interferences of mechanical-quantum superposition is intriguing and challenging.

\section{References}

1. LM Jarvis (2019) Chemical \& Engineering News pp. 41-44.

2. G Villani (2020) A Time-Dependent Quantum Approach to Allostery and a Comparison with Light-Harvesting in Photosynthetic Phenomenon, Front Mol Biosci 7: 156

3. B Buchli, SA Waldauer, R Walser, ML Donten, R Pfister, et al. (2013) Kinetic response of a photoperturbed allosteric protein. Proc Natl Acad Sci U S A. 110: 11725-11730.

4. F Kolodziej, TTan, DE Koshland (1996) Producing Positive, Negative, and No Cooperativity by Mutations at a Single Residue Located at the Subunit Interface in the Aspartate Receptor of Salmonella Typhimurium. Biochemistry 35(47): 14782-14792. 